\title{
Genetic estimates and diversity study in Sesame(Sesamum indicum $\mathbf{L}$.)
}

\author{
Adil Iqbal, Rumana Akhtar, Tamina Begum and Tapash Dasgupta \\ Department of Genetics and Plant Breeding, Institute of Agricultural Science, University of Calcutta, \\ Kolkata-700019, India
}

\begin{abstract}
Genetic estimates and diversity of 33 genotypes sesame representing different eco-geographical regions were studied for number of morphological characters. Wide variation in plant habit (plant height and branching pattern), number of capsules per plant, number of seeds per capsule, mean seed weight, and yield per plant was recorded. The estimates of genotypic coefficient of variation $(G C V)$ were less than its corresponding estimates of PCV for seed yield and yield component, exhibiting consistency in environmental interaction for character expression. Capsule per plant exhibited high heritability coupled with good genetic advance indicating that additive genetic effect was more prevalent in this trait. Correlation study at phenotypic level showed that seed yield per plant was significantly and positive associated with plant height, number of branches per plant, number of capsule per plant and capsule length. So selection for high no of capsule per plant, capsule length and no of branches per plant will lead towards high yield. The genotypes were further grouped through multivariate analysis based on 8 traits. The genotypes constituted three major clusters. Origin of genotypes did not play a significant role in constitution of clusters. Selection of parents based on parents belonging to different cluster would like to produce more desirable segregants.
\end{abstract}

Keywords: Cluster, Correlation, Genetic variability, Heritability and Sesame

\section{Introduction}

Sesame is one of the most ancient and important oilseed crops grown from ancient times. It was cultivated and domesticated on the Indian subcontinent during Harappan and Anatolian eras over 4,000 yrs ago. (Bedigian and Van der Mesen, 2003). Due to the stability of its healthy oil, easiness of extraction and resistance to drought, sesame was popular in the ancient world, Sesame is considered as a nutritious oilseed crop being rich source of protein $(18-25 \%)$, carbohydrate $(13.5 \%)$, minerals and polyunsaturated fatty acid (Bedigian et al. 1986). Sesame oil is favoured as a media of cooking by Indians and Africans. Presence of sesamol, a unique anti-oxidant and more poly-unsaturated fatty acid such as oleic acid (43\%), linoleic acid (35\%), palmitic acid (11\%) and stearic acid (7\%), have made it to 'queen of oilseed crop' (Ashri 1989; Fukuda et al. 1986). Sesame ranks fifth for important edible oil crop in India after groundnut, rapeseed-mustard, sunflower and soybean. (www.agricoop.nic.in). India holds top position in the world in sesame-acreage (24\%) and contribution in export (40\%) (FAO Statistics Division, 2012) Raikwar and Srivastva, (2013). Productivity of sesame in India is low compared to other sesame producing countries. Improvement in productivity will definitely boost the oil crop market of India and other ancillary industries. One of the simple approach to improve production of any crop is to boost up productivity, Genetic up gradation of any crop depends primarily on utilization of existing genetic resource. A large germplasm resource is always favoured in plant breeding program as many desirable traits may obviously remain in the population which may exploit breeding program. Study on genetic diversity comparatively limited in sesame. Several genetic parameters, such as, phenotypic and genotypic coefficient of variation (PCV and GCV), heritability and genetic gain help to assess genetic diversity of experimental materials.

Multivariate analysis is an important biometric technique when different quantitative traits are usually pooled up together to reach towards a conclusive outcome of diversity. Multivariate analysis is often used in selection of parents for hybridization program in different crops like blackgram, (Dasgupta and Das, 1984,1991), horsegram ( Dasgupta et al 2005), mustard (Pandey et al 1984) and sesame (Tripathi et al 2013, Akbar et al 2011). The present study has been conducted to assess the diversity genotypes in sesame following different genetic estimate and multivariate analysis.

\section{Material \& Method}

33 sesame genotypes were collected from diverse eco-geographical regions of India and abroad. The genotypes were grown in summer season following Randomized Block Design (RBD) with 3 replications having $10 \mathrm{~cm}$ spacing between plants and $40 \mathrm{~cm}$ between rows at Agricultural Experimental Farm, University of Calcutta, Baruipur in 2015. The farm is situated at an elevation of 10 meter above sea level, at approximately $22^{0} 51^{\prime} \mathrm{N}$ latitude and $88^{\circ} 24^{\prime} \mathrm{E}$ longitude. The N: P: K fertilizers were applied at the rate 50:25:25 as basal dose 
during final soil preparation. Manganese sulphate at the rate of $5 \mathrm{Kg}$ per hectare was also given as basal. Normal culture practices were followed during cultivation and irrigations were applied whenever the soil becomes very dry. Statistical cluster analysis of 33 genotypes with respect to morphological characters was done by Average Linkage Between Groups (in other words UPGMA) Method. The divergence between accessions was evaluated using a Euclidean distance dissimilarity matrix. Euclidian distance between lines were calculated and UPGMA cluster analysis was performed with the help of IBM-SPSS software (Version: 16.0) for dendrogram formation

\section{Result \& discussion}

Eight morphological characters were studied and ANOVA (Analysis of Variance) revealed that genotypes were significantly different from each other indicating high diversity among the genotypes.

Phenotypic coefficients of variation exhibited marginal higher values but maintained a close relation with genotypic coefficients of variation for all the traits. Highest coefficients of variation (phenotypic and genotypic) were exhibited by capsule per plant, seed yield/plant ( Table 2) Sumathi and Murlidharan (2010), Parameshwarappa et al., (2009) and Sudhakar et al. (2007), and number of primary branches/plant similar finding was reported earlier Solanki and Gupta, (2003), Sudhakar et al. (2007), Saha et al. (2012), Gidey et al., (2013), Iqbal and Dasgupta, (2015), in sesame. High GCV and PCV for capsule/plant, seed yield/plant and number of primary branches/plant suggest reasonably high variability in the studied materials and this would obviously help in upgrading the genotypes by simple selection. Heritability in broad sense was highesr for 1000 seed weight followed by capsule per plant and seed yield/plant. Moderately heritability was observed for capsule length, plant height and days to $50 \%$ flowering on the contrary days to maturity exhibits very low heritability. Information on heritability of any traits aids to selection as traits with high heritability would like to exhibit similar character experrsion consistently over years. High heritability with high GCV of any trait is favourable combination for genetic up gradation of any trait as better estimates simultaneously in two biometrical parameter of any trait indicate that the genetic control of the trait is additive in nature( Pham et al 2011). The trait Capsule/plant followed by seed yield/plant showed such ideal combination in genetic estimates. High heritability with genetic advance of any trait point out that the trait is under additive genetic control. In self pollinated crop additive gene effect is more desirable than non-additive genetic effect. Capsule/plant showed high GA, high heritability and also GCV. So, capsule/plant exhibits innate potentiality for genetic improvement in sesame. Such combination was not observed in any other trait. Two other traits namely 1000 seed weight and seed yield/plant were characterized by high heritability with moderate genetic advance, moderate heritability with low genetic advance were found for plant height, branches per plant and days to flowering. The results confirm previous finding of Reddy et al (2001), Iqbal and Dasgupta (2015).

Correlation study ( genotypic and phenotypic ) were showed that plant height, number of branches per plant, no of capsule per plant and capsule length were positively and significantly correlated with seed yield per plant ( Table 3). The results are in concurrence with the results of Uzun and Cagirgan (2001), and Sumathi et al. (2007) Subramanian and Subramanian (1990). Thus selection of any of these characters would lead to the improvement of seed yield/plant. On the contrary, 1000 seed weight showed negative relationship with seed yield parsaeian et al. (2010). .

A few inter- relationships showed significant and positive correlation namely plant height and number of branches/plant, number of capsule/plant and number of primary branches/plant, Plant height and number of capsule/plant, Capsule length and number of capsule/plant, number of capsules per plant and seed yield per plant. Negative interrelationship were found between days to 50\% Flowering and 1000 seed weight, days to $50 \%$ flowering and capsule length. Days to maturity also had negative effect on capsule length and 1000 seed weight though the effect was not significant.

Combining all traits, selection for plant height would not only improve seed yield/plant but also will improve capsules/plant and branches/plant through correlated response. However in intensive cropping system the breeders are interested for early maturity though delayed maturity improves seed size.

Improvement of the trait capsule/plant would also improve plant height, branches/plant, capsule length and ultimately seed yield/plant. So, capsules/plant was the most desired trait for improvement of several traits and also seed yield/plant. It is interesting to note that capsules/plant had additive genetic control with high GCV, high GA and high heritability.

All 33 genotypes were further analyzed by SPSS for grouping. Three distinct clusters were found. (Figure 1 and Table 4). Cluster I comprised of 23 genotype where as cluster II consisted of 6 genotypes and cluster III consisted 4 genotypes. Four distinct sub-clusters were identified for cluster I. The sub- clusters IA, IB, IC and ID contained 8, 7, 2 and 6 genotypes respectively. Cluster IA has been further grouped into two distinct sub clusters IA-i and IA-ii comprising 4 genotypes and 2 genotypes each. The maximum Euclidean distance recorded between RT-346 and EC-303435(4) followed by EC-204704 and RT-346, IC-20477 and RT346. Desirable segregates are expected if crossing is done between genotypes with high dissimilarities coefficient. 
An investigation of the cluster composition revealed that each of the two clusters consisted of varieties belonging to different origin, i.e. from different states of India and also from different countries.This indicate that genetic divergence of genotypes is independent of geographic origin. Similar finding was also reported Banerjee and Kole (2009), Kandamoorthy and Govindarasu (2005), Banumathy et al. (2010) and Saha et al (2012).

\section{Conclusion}

Sesame genotypes showed considerable genetic variability and divergence. It was found that Additive genetic effect was more prevalent for Capsule per plant. Correlation study at phenotypic level showed that seed yield per plant was significantly and positive associated with plant height, number of branches per plant, number of capsule per plant and capsule length, so selection for high no of capsule per plant, capsule length and no of branches per plant will lead towards high yield. The cluster analysis helped in grouping the genotypes into different clusters having specific characteristic traits which may be helpful in selecting parents for future breeding programs. Crossing between the genotypes RT-346 ,EC-303435(4), EC-204704 and IC-20477 would most likely express considerable amount of heterosis in F1 generation and also provide a wide spectrum of recombinants in segregating generations. Grouping of genotypes based on multivariate analysis was independent of origin of cultivars. The conventional assumption that selecting genotypes of different geographical origin will maximize the diversity available to a breeding project does not follow in sesame.

\section{Acknowledgement}

We acknowledge the university grants commission for providing the financial support to carry out the study under UGC Major Project on sesame title Genetic diversity among genotypes and molecular linkage map construction in sesame (Sesamum indicum L.) UGC Reference No. F. 42-721/2013(SR)

Table 1 : List of Genotypes

\begin{tabular}{|c|c|c|c|c|c|}
\hline Serial No. & Name of genotypes & Origin & Serial No. & Name of genotypes & Origin \\
\hline 1 & Savitri & Malda & 18 & TKG-352 & Tikamgarh \\
\hline 2 & Rama & Malda & 19 & TKG-22 & Tikamgarh \\
\hline 3 & Saheb & West Bengal & 20 & IC-20477 & India \\
\hline 4 & Osc-207 & Odisha & 21 & IC-26230 & India \\
\hline 5 & Cums-20 & West Bengal & 22 & IC-141464 & India \\
\hline 6 & DSS-09 & Karnatak & 23 & EC-182832(26) & Bulgaria \\
\hline 7 & B-76 & South 24 parganas & 24 & EC-334988(3) & Bulgaria \\
\hline 8 & $\mathrm{~V}-13$ & West Bengal & 25 & EC-303435(4) & Bulgaria \\
\hline 9 & $\mathrm{~V}-15$ & Hoogly & 26 & EC-161492-A & Bulgaria \\
\hline 10 & CST-2001 & West Bengal & 27 & EC-164966(50) & USA \\
\hline 11 & Haveri & West Bengal & 28 & EC-204704 & USA \\
\hline 12 & Utawadia & West Bengal & 29 & EC-41923(B) & USA \\
\hline 13 & Cums-11 & West Bengal & 30 & EC-303433 & USA \\
\hline 14 & Cums-9 & West Bengal & 31 & EC-303442(32) & Bulgaria \\
\hline 15 & VRI-1 & Tamil Nadu & 32 & EC-100043-A & Bulgaria \\
\hline 16 & RT-346 & Rajasthan & 33 & EC-100049(3) & Bulgaria \\
\hline 17 & GT-Black & Gujarat & & & \\
\hline
\end{tabular}

Table.2. Estimates of variability, Heritability and Genetic Advance in sesame

\begin{tabular}{|l|l|l|l|l|l|l|l|l|}
\hline & $\begin{array}{l}50 \% \\
\text { Flowering }\end{array}$ & $\begin{array}{l}\text { Days to } \\
\text { Maturity }\end{array}$ & $\begin{array}{l}1000 \text { Seed } \\
\text { Weight }\end{array}$ & Plant Height & $\begin{array}{l}\text { Branches } \\
\text { per Plant }\end{array}$ & $\begin{array}{l}\text { Capsule } \\
\text { Length }\end{array}$ & $\begin{array}{l}\text { Capsule per } \\
\text { Plant }\end{array}$ & $\begin{array}{l}\text { Seed yield } \\
\text { Per Plant }\end{array}$ \\
\hline GCV & 5.76 & 1.97 & 26.11 & 14.64 & 29.31 & 13.15 & 62.56 \\
\hline PCV & 7.92 & 4.34 & 26.30 & 20.73 & 42.21 & 16.34 & 67.70 \\
\hline H\% & 52.88 & 20.61 & 98.57 & 49.9 & 48.21 & 64.79 & 85.39 & 64.37 \\
\hline GA & 2.69 & 0.74 & 1.27 & 13.57 & 1.63 & 0.38 & 72.09 & 2.14 \\
\hline Mean & 42.98 & 88.899 & $2.479(\mathrm{gm})$ & $90.111(\mathrm{~cm})$ & 5.596 & $2.176(\mathrm{~cm})$ & 65.576 & $4.383(\mathrm{gm})$ \\
\hline Range & $36.33-48.00$ & $83.00-94.67$ & $1.64-3.13$ & $70.33-121.67$ & $2.67-8.00$ & $1.57-3.07$ & $\begin{array}{l}29.00- \\
193.00\end{array}$ & $4.04-8.22$ \\
\hline
\end{tabular}


Genetic estimates and diversity study in Sesame(Sesamum indicum L.)

Table- 3: Phenotypic Correlation Matrix

\begin{tabular}{|c|c|c|c|c|c|c|c|c|}
\hline & $\begin{array}{l}\mathbf{5 0 \%} \\
\text { flowering }\end{array}$ & $\begin{array}{l}\text { Days to } \\
\text { maturity }\end{array}$ & $\begin{array}{l}\text { 1000 Seed } \\
\text { weight }\end{array}$ & $\begin{array}{l}\text { Plant } \\
\text { Height }\end{array}$ & $\begin{array}{l}\text { Branches } \\
\text { per Plant }\end{array}$ & $\begin{array}{l}\text { Capsule } \\
\text { Length }\end{array}$ & $\begin{array}{l}\text { Capsule } \\
\text { per Plant }\end{array}$ & $\begin{array}{l}\text { Seed yield } \\
\text { Per Plant }\end{array}$ \\
\hline $50 \%$ flowering & 1.000 & & & & & & & \\
\hline $\begin{array}{ll}\text { Days } & \text { to } \\
\text { maturity } & \end{array}$ & 0.285 & 1.000 & & & & & & \\
\hline $\begin{array}{ll}1000 & \text { Seed } \\
\text { weight } & \end{array}$ & -0.120 & 0.049 & 1.000 & & & & & \\
\hline Plant Height & $0.353^{*}$ & 0.240 & -0.056 & 1.000 & & & & \\
\hline $\begin{array}{l}\text { Branches per } \\
\text { Plant }\end{array}$ & 0.149 & -0.050 & 0.058 & 0.151 & 1.000 & & & \\
\hline Capsule Length & 0.234 & -0.138 & -0.153 & -0.065 & 0.044 & 1.000 & & \\
\hline $\begin{array}{l}\text { Capsule per } \\
\text { Plant }\end{array}$ & 0.232 & 0.052 & -0.026 & 0.080 & $0.646^{* *}$ & 0.088 & 1.000 & \\
\hline $\begin{array}{l}\text { Seed yield Per } \\
\text { Plant }\end{array}$ & $0.613^{* *}$ & 0.074 & -0.070 & $0.461^{* *}$ & $0.435^{* *}$ & $0.414^{* * *}$ & $0.536^{* *}$ & 1.000 \\
\hline
\end{tabular}

${ }^{*}$ and ${ }^{* *}$ : significant at $5 \%$ and $1 \%$ level respectively

Table 4: Cluster composition of 33 genotypes

\begin{tabular}{|l|l|l|l|l|}
\hline CLUSTER & \multicolumn{2}{|l|}{ SUB CLUSTER } & $\begin{array}{l}\text { NUMBERS OF } \\
\text { GENOTYPES }\end{array}$ & ACCESSION NUMBER \\
\hline CLUSTER-I & A & i & 8 & $\begin{array}{l}\text { DSS-09, EC-100043-A, V-13, EC-303442(32), IC-141464, EC- } \\
303433, \text { Cums-9, Rama }\end{array}$ \\
\cline { 3 - 5 } & & 7 & $\begin{array}{l}\text { IC-26230, EC-161492-A, Haveri, EC-164966(50), Saheb, EC- } \\
334988(3), \text { EC-41923(B), }\end{array}$ \\
\cline { 2 - 5 } & ii & 7 & EC-303435(4), EC-204704 \\
\cline { 2 - 5 } & B & 2 & V-15, GT-Black, B-76, CST-2001, EC-182832(26), EC-100049(3) \\
\cline { 2 - 5 } & C & 6 & Osc-207, Cums-11, VR-I, Utawadia \\
\cline { 2 - 5 } & D & 4 & Cums-20, IC-20477 \\
\hline CLUSTER- II & & 2 & Savitri, TKG-352, TKG-22, RT-346 \\
\hline CLUSTER- III & & 4 &
\end{tabular}

Table 5: Euclidean distance between 33 genotypes

\begin{tabular}{|c|c|c|c|c|c|c|c|c|c|c|c|c|c|c|c|c|c|c|c|c|c|c|c|c|c|c|c|c|c|c|c|c|c|}
\hline & t & I & 1 & 4 & I & 1 & I & 1 & 2 & 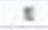 & $t$ & $E$ & 5 & $z$ & I & 8 & F & $t$ & $\leqslant$ & $z$ & $z$ & 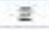 & 8 & 3 & 5 & $x$ & $z$ & $\gamma$ & $\underline{z}$ & $z$ & 2 & $z$ & $z$ \\
\hline$t$ & 1 & $t a$ & $\bar{z}$ & 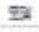 & 66 & EII & $z$ & ex & $\pi$ & 32 & III & 28 & EE & BEE & e & EII & $\varepsilon z$ & 28 & 38 & $\$ 3$ & $\mathrm{es}$ & $n$ & $\mathrm{~ns}$ & ES & हैँ & $5=$ & $\cong$ & a & $=1$ & $e x$ & ra & xis & az \\
\hline 1 & & 1 & 3.2 & $\operatorname{sa}$ & 32 & 28 & et & $\varepsilon$ & III & $x_{1}$ & 17 & of & $y z$ & $\leq 8$ & $\leq 2$ & 232 & 25 & $\pi$ & $\operatorname{ser}$ & 52 & $\underline{I I}$ & $\mathrm{tz}$ & 53 & $a$ & 28 & 30 & 15 & zs & 22 & 5 & $y z$ & 328 & 23 \\
\hline 2 & & & 1 & ex & 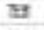 & $x$ & ase & 81 & $y$ & w & $\mathrm{v}$ & It & ser & $\sqrt{13}$ & $\pi s$ & 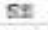 & बII & $\pm x$ & 28 & 85 & $\mathrm{res}$ & $\equiv 3$ & खN & 2 & xe & $\varepsilon$ & $n$ & 58 & II & Is & 35 & II & 20 \\
\hline 4 & & & & $t$ & 23 & $x$ a & se & 21 & $x 2$ & SII & za & \pm 2 & हе & zas & $y$ & Ke & za & er & ses & $\mathrm{m}$ & It & 32 & $m x$ & es & 38 & $E 5$ & $\Sigma 8$ & Ir & $20 x$ & at & $\mathrm{n}$ & $a z$ & 32 \\
\hline 5 & & & & & I & 35 & tot & \$I & 28 & $\mathrm{zI}$ & $w$ & TE & $z$ & ef & 53 & xal & ER & $m$ & ex & $\pi$ & 35 & xe & Iff & \pm & $\mathbb{E}$ & $\mathrm{min}$ & ex & 25 & 10 & 28 & II & $r z$ & os \\
\hline i & & & & & & $t$ & $3 x$ & tu & $t$ & 21 & II & $\mathrm{ex}$ & $2 Z$ & $\leq 8$ & \pm 0 & $a$ & $\varepsilon \varepsilon$ & $\pi R$ & $\mathrm{Es}$ & Is & af & as & 288 & I5 & $E s$ & $\mathrm{ar}$ & as & $E \mathscr{F}$ & 23 & 32 & ac & $s e$ & to \\
\hline$T$ & & & & & & & I & In & $a$ & $2 x$ & EIE & $\mathrm{egr}$ & ge & 2) & ext: & 58 & t5f & $a z$ & $\mathrm{Ef}$ & 58 & $\mathrm{BE}$ & $x=$ & IE & es & 렬 & 3 & $\boldsymbol{E}$ & क्रत & 38 & $X E$ & In & $20 \mathrm{r}$ & Ir \\
\hline$t$ & & & & & & & & $t$ & $\mathrm{sz}$ & $x$ & $z$ & est & $\mathbf{p}$ & ta & 23 & ex & 28 & $\mathrm{ke}$ & $8 x$ & 52 & $\mathrm{IE}$ & If & $\mathrm{m}$ & 38 & 27 & 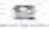 & sil & 2 & $x z$ & เร & 12 & $\mathrm{ax}$ & 92 \\
\hline$z$ & & & & & & & & & t & $2 t$ & III & $5 \pi$ & 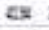 & zxf & $\| \equiv$ & 2 & ter & $5 x$ & $\mathrm{ex}$ & 98 & 32 & $8 E$ & $a 2$ & 28 & 21 & 321 & $3 x$ & 201 & st & \pm & tE & 七] & sf \\
\hline 1 & & & & & & & & & & $t$ & es & \pm & Et & 35 & 23 & $t x$ & 28 & 8 & If & s: & 33 & $x$ & 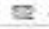 & sa & 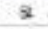 & 50 & $\mu$ & Es & $\mathrm{m} 18$ & 21 & 23 & 21 & $2 x$ \\
\hline$t$ & & & & & & & & & & & I & EI & 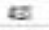 & $t 2$ & 23 & IfI & es & 23 & 15 & 80 & 88 & 25 & $E$ & az & Ex & se & Es & sI & 28 & tes & 23 & 321 & 3 \\
\hline 2 & & & & & & & & & & & & $t$ & $\mathbb{Z e}$ & $\mathrm{mE}$ & 32 & ex & SI & ex & $\mathrm{zx}$ & in & nt & $\mathbb{2}$ & $\mathbf{y}$ & FIs & st & $\mathrm{En}$ & $\approx x$ & an & $\mathrm{fz}$ & se & xal2 & 25 & es \\
\hline$=$ & & & & & & & & & & & & & t & tw & E & 285 & 62 & $z x$ & $\mathbf{y}$ & 12 & $\geq 2$ & IE & II & 5 & zr & 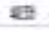 & ex & es & $a t$ & $2 x$ & eat & 86 & $x$ \\
\hline x & & & & & & & & & & & & & & $t$ & as & 252 & 27 & $\pi$ & 32 & aff & $\varepsilon \approx$ & 32 & $\pi 2$ & $\mathbb{E}$ & 28 & af & $\mathrm{tz}$ & $\approx$ & 8 & IE & 23 & 1al & xat \\
\hline 5 & & & & & & & & & & & & & & & $8:$ & $a$ & $\Leftrightarrow$ & sE & $2 x$ & $E x$ & 15 & $\geq$ & $\mathrm{as}$ & III & EE & ह5 & 28 & E2 & $w$ & re & $2 E$ & v & $m$ \\
\hline 1 & & & & & & & & & & & & & & & & t: & 23 & 22 & 25 & $w$ & $5 x$ & $a$ & $\mathrm{rab}$ & se & $B$ & $\operatorname{sat}$ & $20 x$ & ESE & 98 & an & eE & 20 & Eat \\
\hline$\pi$ & & & & & & & & & & & & & & & & & 8 & II & Ex & ser & EI & tax & 20 & $\boldsymbol{E}$ & RE & $z$ & Eal & छा & EF & $\approx$ & $\underline{x E}$ & 58 & y \\
\hline 1 & & & & & & & & & & & & & & & & & & 6 & se & $\cong$ & $=8$ & $\pi$ & 251 & $2 x$ & $=$ & $\geq z$ & $a$ & 526 & $2 \pi$ & $\varepsilon t$ & $\mathrm{rat}$ & $\mathrm{seg}$ & ve \\
\hline 2 & & & & & & & & & & & & & & & & & & & 8 & 52 & $2 x$ & IIT & $\boldsymbol{x}$ & ast & $\pi 2$ & as & eu & 3 & 35 & ts & es & tes & 52 \\
\hline$y$ & & & & & & & & & & & & & & & & & & & & 6 & $t$ & 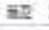 & $\operatorname{se}$ & Ex & $\mathrm{nat}$ & $\pi z$ & $\mathrm{sr}$ & $w x$ & $\bar{\omega} \bar{x}$ & 吸 & $E 2$ & 23 & ax \\
\hline$z$ & & & & & & & & & & & & & & & & & & & & & 18 & 20 & of & ss & 21 & $c 2$ & Et & $\approx$ & 25 & 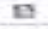 & $\mathbf{z}$ & $2 z$ & 80 \\
\hline$\equiv$ & & & & & & & & & & & & & & & & & & & & & & 8 & 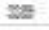 & $\mathrm{se}$ & ss & $\mathbf{E}$ & 25 & 28 & $3 x$ & 25 & 12 & $\leq \varepsilon$ & $z x$ \\
\hline$\equiv$ & & & & & & & & & & & & & & & & & & & & & & & 1 & Ea & $\overrightarrow{x I}$ & es & $a z$ & $\mathbb{a}$ & $3 x$ & $2 \pi$ & $3 z$ & II & $\approx$ \\
\hline$x$ & & & & & & & & & & & & & & & & & & & & & & & & 1 & 23 & as & $3 \mathrm{si}$ & 22 & 28 & $\varepsilon$ & 321 & $\mathrm{ss}$ & zI \\
\hline 8 & & & & & & & & & & & & & & & & & & & & & & & & & 1 & 21 & $t z$ & 12 & 5 & sx & E & $\mathbf{M}$ & $5 x$ \\
\hline 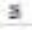 & & & & & & & & & & & & & & & & & & & & & & & & & & 1 & $\mathrm{st}$ & 25 & 23 & 28 & $\mathrm{n}$ & $3 x$ & $\mathrm{~s}$ \\
\hline$a$ & & & & & & & & & & & & & & & & & & & & & & & & & & & 1 & $z 2$ & $v$ & 28 & za & 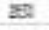 & $\mathrm{s}$ \\
\hline a & & & & & & & & & & & & & & & & & & & & & & & & & & & & 1 & E & se & $\mathbb{E}$ & $\$ 3$ & $\mathbf{Z z}$ \\
\hline$z$ & & & & & & & & & & & & & & & & & & & & & & & & & & & & & 1 & 28 & $2 \mathrm{si}$ & 22 & $\mathrm{gat}$ \\
\hline 2 & & & & & & & & & & & & & & & & & & & & & & & & & & & & & & 1 & 201 & 16 & $2 \pi$ \\
\hline$x$ & & & & & & & & & & & & & & & & & & & & & & & & & & & & & & & 1 & w & $\mathbf{v}$ \\
\hline 2 & & & & & & & & & & & & & & & & & & & & & & & & & & & & & & & & 1 & 38 \\
\hline$z$ & & & & & & & & & & & & & & & & & & & & & & & & & & & & & & & & & 1 \\
\hline
\end{tabular}




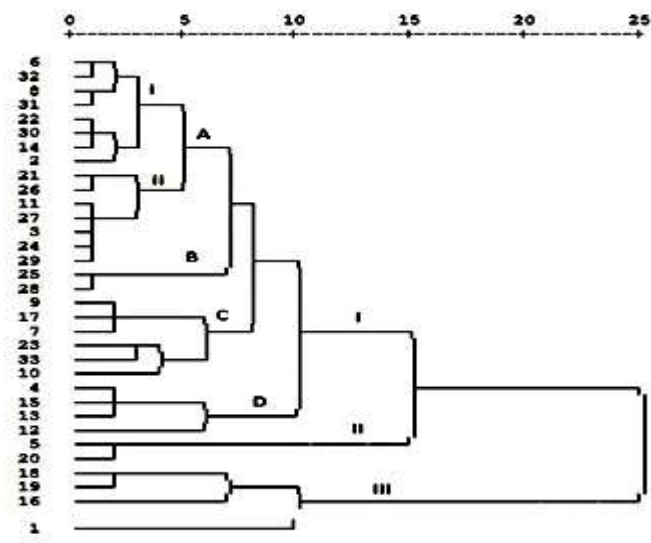

Fig: 1 Dendrogram showing the distribution of 33 genotypes in clusters

\section{References}

[1]. D Bedigian and L. J. G. van der Maesen, Slimy leaves and oily seeds: Distribution and use of Sesamum spp. and Ceratothecasesamoides (Pedaliaceae) in Africa, 2003.

[2]. D Bedigian, CA Smith and JR Harlan, Patterns of morphological variation in Sesamum indicum. Economic Botany 40: 1986, 353365 .

[3]. Amram Ashri, "Sesame breeding." Plant breeding reviews 16, 1998, 179-228.

[4]. Fukuda, Y., et al. "Contribution of lignan analogues to antioxidative activity of refined unroasted sesame seed oil." Journal of the American Oil Chemists' Society 63(8) 1986, 1027-1031.

[5]. FAOSTAT, (2012).Faostat.Fao.org.

[6]. R. S Raikwar, and P. Srivastva, Productivity enhancement of sesame (Sesamum indicum L.) through improved production technologies, African Journal of Agricultural Research 8(47), 2013, 6073-6078.

[7]. T Dasgupta and P K Das, Multivariate analysis and selection of parents for hybridization in blackgram, Philippin Agriculturist. 44(1), 1984, 86-92

[8]. T Dasgupta and P K Das, Genetic divergence in blackgram, Ind.J. Agri. Res 25(1), 1991,7-13

[9]. T Dasgupta, K Mukerjee, B Roychoudhury and D Nath, Genetic divergence in horse gram leg , , Ind.J. Agri. Res. 28(3), 2005,166171

[10]. K. P Pandey, R. Singh, and C. P. M. Tripathi. "Functional response of Diaeretiella rapae (M'Intosh)(Hym., Aphidiidae), a parasitoid of the mustard aphid Lipaphis erysimi Kalt.(Hom., Aphididae)." Zeitschrift für Angewandte Entomologie 98.(1-5) 1984, 321-327.

[11]. F Akbar, MA Rabbani, , ZK Shinwari, and SJ Khan, Genetic diversity in sesame (Sesamum indicum landraces based on qualitative and quantitative traits, Pak. J. Bot 43(6), 2011,2737-2744.

[12]. A Tripath, B Rajani, R. P. Ahirwal, S Paroha., R Sahu and A Ranganatha,. Study on genetic divergence in sesame (Sesamum indicum L.) germplasm based on morphological and quality traits. The Bioscan, 8(4), 2013;1387-1391.

[13]. P. Sumathi and V Murlidharan, Analysis of genetic variability, association and path analysis in the hybrids of sesame (Sesamum indicum L.), Tropical Agricultural Research and Extension. 13(3), 2010, 63-67.

[14]. S. G Parameshwarappa, M. G Palakshappa, P. M Salimath, and K. G Parameshwarappa, Studies on genetic variability and character association in germplasms collection of sesame (Sesamum indicum L.), Karnataka Journal of Agricultural Sciences, 22(2). 2010.

[15]. N. Sudhakar, O Sridevi and P Salimath, Variability and character association analysis in sesame (Sesamum indicum L.). Journal of Oilseeds Research. 24(1), 2007, 56-58.

[16]. Z. S Solanki,. and D Gupta, Variability and character association among quantitative characters of sesame, J. Oilseeds Res. 20, 2003, 276-277.

[17]. S Saha, , T Begum and T Dasgupta, Analysis of genotypic diversity in sesame based on morphological and agronomic traits. Conference on international research on food Security, natural resourcemanagement and rural development organised by GeorgAugust Universität Göttingen and University of Kassel-Witzenhausen, Germany during Sept 2012

[18]. Y. T Gidey, S. A Kebede,. and G. T. Gashawbeza, Assessment of genetic variability, genetic advance, correlation and path analysis for morphological traits in sesame genotypes, Int. J. Plant Breeding and Gent. 7(1), 2013,21-34.

[19]. A Iqbal and T Dasgupta, Genetic Estimates of Morphological Traits and Phenotypic Diversity in Core Collections of Sesame (Sesamum indicum L.) Indian Agriculurist, Vol.5, 2015, 61-69.

[20]. T. D Pham, M. Geleta, T. M Bui, T. C Bui, A Merker,. and A. S Carlsson, Comparative analysis of genetic diversity of sesame (SesamumindicumL.) from Vietnam and Cambodia using agro-morphological, Hereditas ,148(1), 2011, 28-35.

[21]. P. A.V Reddy, M. R Sekhar, A. R. G Rangnatha and A Dhanraj, Genetic variability and heritability for seed yield and its components in Sesame. J. Oilseeds Res., 18, 2001, 173-175.

[22]. B Uzun, \& M. İ. Çağırgan, Comparison of determinate and indeterminate lines of sesame for agronomic traits. Field crops research, 96(1), 2006,13-18.

[23]. S Subramanian, \& M. Subramanian, Correlation studies and path coefficient analysis in sesame (Sesamum indicum L.), Journal of agronomy and crop science, 173(3-4), 1994, 241-248.

[24]. M Parsaeian, A. Mirlohi, and G. Saeidi, Study of genetic variation in sesame (Sesamum indicum L.) using agro-morphological traits and ISSR markers, Russian journal of genetics 47(3), 2011, 314-321.

[25]. P. P. Banerjee and P. C. Kole, Analysis of genetic architecture for some physiological characters in sesame (Sesamum indicum L.). Euphytica, 168(1), 2009, 11-22.

[26]. S Kandamoorthy and R Govindarasu, Genetic divergence in extra early rice (Oryza sativa L.) under two culture systems, Indian J. Genet. (65) 2005, 43-44.

[27]. S Banumathy, R Manimaran, A Sheeba, N Manivannan, B Ramya, D Kumar and GV Ramasubramanian, Genetic diversity analysis of rice germplasm lines for yield attributing traits. Electronic Journal of Plant Breeding (1)2010, 500-504. 